\title{
Challenge - nanotechnology in engineering
}

\section{Introduction}

Nanostructured materials are very interesting and important systems, challenging science and technological application in engineering and medicine. In general, materials containing grains, clusters, foils or filaments of dimensions lower than $10 \mathrm{~nm}$ are considered nanostructured materials, because their properties differ from the extended solids. These materials have been extensively studied in the last year's Figure 1 since the small dimensions of primary building present high surface/ volume ratios resulting in special mechanical, optical, electronic and magnetic properties; Nanoparticles of transition metals exhibit special behaviors due to the effects related by reducing sizes. In fact, these materials modify the electronic structure, offering new developments with different characteristics for reactivity and effectiveness in different processes. These behaviors can be associated to the effect of surface, such as superfluity and differentiated dynamic sintering

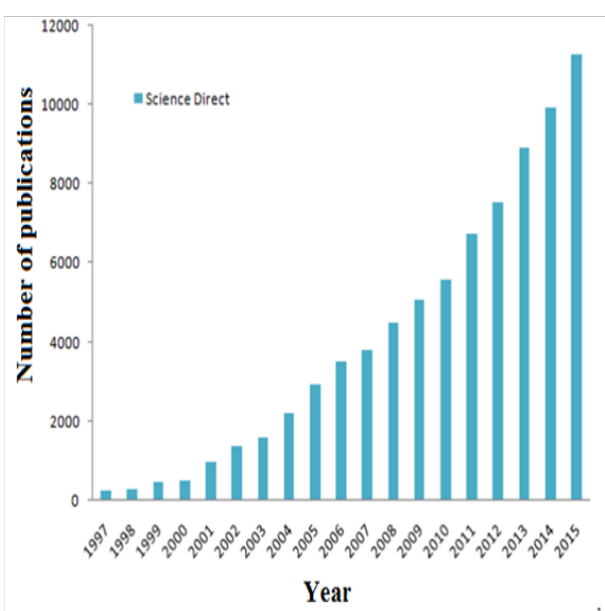

Figure I Publication increasing every year.

In engineering we must stand out the properties associated to different morphologies, where the activity and selectivity are strongly affected by the form and particle sizes and, in the case of crystallite phases, on the direction and exposure to different faces. The reactions influenced by these factors are well known as structure sensitive or insensitive reactions. Therefore, small sizes and high surface/volume ratios provide excellent reactivity, when compared to the bulk materials. There are several natural and synthesized materials with specific properties, which cover practically all applied engineering and medicine. A general view of applied nanostructure systems is shown in Figure $2{ }^{1}$

\section{Nanostructured materials}

There are several ways leading to synthesize nanostructured materials. The methods may involve preparation of new compounds starting from different precursors, which represents the chemical route or vaporization processes followed by refrigeration of the proper material under controlled conditions.

The catalytic activity of chemical processes depends on the metallic particle sizes. These particles also depend on the preparation
Volume 2 Issue 5 - 2017

Martin Schmal
Department of Chemical Engineering, University of São Paulo,
Brazil

Correspondence: Martin Schmal, Department of Chemical Engineering, University of São Paulo, Brazil, Email schmal@peq.coppe.ufrj.br

Received: February 22, 2017| Published: July 03, 2017

methods and treatment conditions. The sizes and morphologies changed significantly using different stabilizers. Suslick et al. ${ }^{2}$ studied the Fischer-Tropsch Synthesis with $\mathrm{Fe} / \mathrm{SiO}_{2}$ catalysts, synthesized by different methods resulting in different particles sizes. Comparing the activity of the catalysts prepared by impregnation with the prepared by sonochemical method, it shows that the activity for nanoparticles exhibited elevated rates when compared to the larger particles.

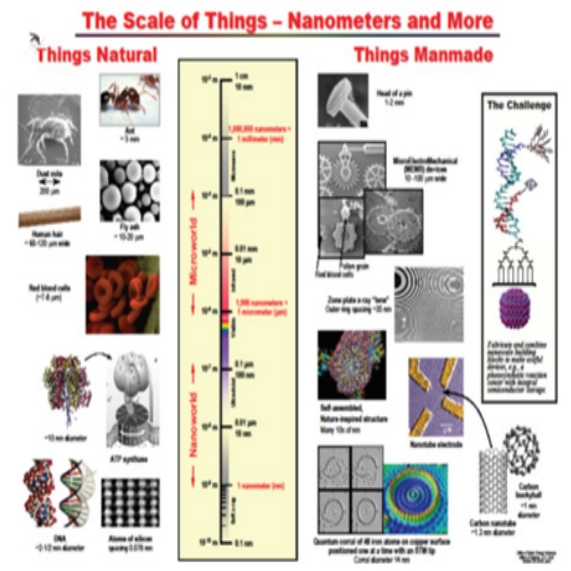

Figure 2 View of applied nanostructure systems

Experiments allow to obtain nanocrystals in a wide range of sizes and shapes presenting active sites that allow to promote diverse chemical reactions. Control at the molecular level about the nature of active centers is important. Thus, different systems were studied and synthesized using colloidal chemical processes. In this way, nanostructures with a very homogeneous distribution were obtained, which depend fundamentally on the methods used, for example, nanotowers, nanocone, nanobottle, nanoflowers, nanoarrows, nanorods, nanowires. Fine films or porous membranes and nanotubes of chemically stable conducting oxides with high surface and functionalized areas were obtained for use in photovoltaic systems, photodetectors, photo-electrochemical, photo-catalysis, and chemical electrodes. ${ }^{3}$ 


\section{Carbon nanotubes (CNT)}

This is an allotropic form of carbon, just like graphite or diamond, discovered in 1991 by S. Iijima. Carbon nanotubes consist of a hollow tubular structure formed by carbon atoms with sp2 hybridization arranged hexagonally, such as a rolled graphene sheet, the ends of which may or may not be closed. The diameter of these tubes is commonly in the range of 2-50 $\mathrm{nm}$. They can be formed by a layer (SWNT) or multilayer (MWNT). Its properties can be summarized as follows: ${ }^{4}$

i. Single layer nanotubes (SWNTs) can be electron conductors or semi-conductors like: arm-chair (conductor), chiral (semi-conductor) or zig-zag (semi-conductor).

ii. Multilayer's (MWNTs) are good electron conductors.

iii. They have great thermal stability (thermal)

As shown in Table 1 the mechanical resistance and thermal conductivity of the single and multiwall nanotubes present significant differences when compared to Steel and Diamond. In fact, these materials are important for many applications in electrical/electronics, optoelectronics, sensors, in particular for electronic devices. ${ }^{4}$ Significant are also the surface areas, which are very high and suitable for chemical processes and adsorption systems, such as, materials for hydrogen storage and catalyst favoring high metal dispersions.

Table I Properties of carbon nanotubes ${ }^{4}$

\begin{tabular}{|c|c|c|c|}
\hline Property & SWNT & MWNT & Comparison \\
\hline Young Modulus (GPa) & 1054 & 1200 & 208 (Steel) \\
\hline $\begin{array}{l}\text { Mechanical Resistance } \\
\text { (GPa) }\end{array}$ & 150 & 150 & 0.4 (Steel) \\
\hline $\begin{array}{l}\text { Thermal conductivity } \\
\left(\mathrm{W} / \mathrm{m}^{2} \mathrm{~K}\right)\end{array}$ & 3000 & 3000 & 2000 (Diamond) \\
\hline Surface Area $\left(\mathrm{m}^{2} / \mathrm{g}\right)$ & $200-400$ & $400-900$ & $\begin{array}{l}1000 \text { (Active } \\
\text { Carbon) }\end{array}$ \\
\hline
\end{tabular}

CNT are chemically inert in acidic or basic media and thus materials which are used as catalyst or carries. They have been used in the decomposition of methane, in oxidation, esterification and hydroxylation reactions. However, the NTC are frequently used as supports impregnated with metallic particles in several reactions, such as hydrogenation, polymerization, ammonia decomposition, oxidation, and finally in Reforming, Partial Oxidation, Prox reactions and Fischer-Tropsch synthesis, among others. The surface needs to be activated or functionalized to create anchor centers for nanoparticles. The CNT proved to be attractive and competitive in catalytic processes due to the combination of its unique morphology and electronic, mechanical, thermal and adsorption properties. ${ }^{5-8}$

\section{Graphenes}

Finally, graphene's are two-dimensional planar atomic layers of type sp2, with dimensions around $34 \mathrm{~nm}$, and are excellent electronic conductors, presenting high mechanical stability, used special use in optical, thermal and catalytic systems. It still presents difficulties for mass production. Graphene oxide contains many functional groups, hydroxides and carbonyls at the corners of the graphene layers. The synthesis of graphene is based on oxidation of graphite and reduction of the graphite oxide, using chemical or thermal methodologies. This procedure was invented by Hummers ${ }^{9}$ and improved, as described Park et al. ${ }^{10,11}$

Graphenes are frequently used as supports for producing catalyst with high surface area. They also present strong interaction with the metals or oxide, with excellent electronic properties, which allow improving the adsorption-desorption- reaction process.

Some researchers have found good results through the use of graphene as a support for monometallic and bimetallic catalysts Figure 3. One interesting example is the production of syntheses gas and fuels in the reforming and Fischer- catalysts supported on different materials. Karimi et al. ${ }^{13}$ compared metals supported on graphenes, CNT and Silica. The $\mathrm{Co} / \mathrm{Graphene}$ is a promising catalyst for this reaction and other related processes.

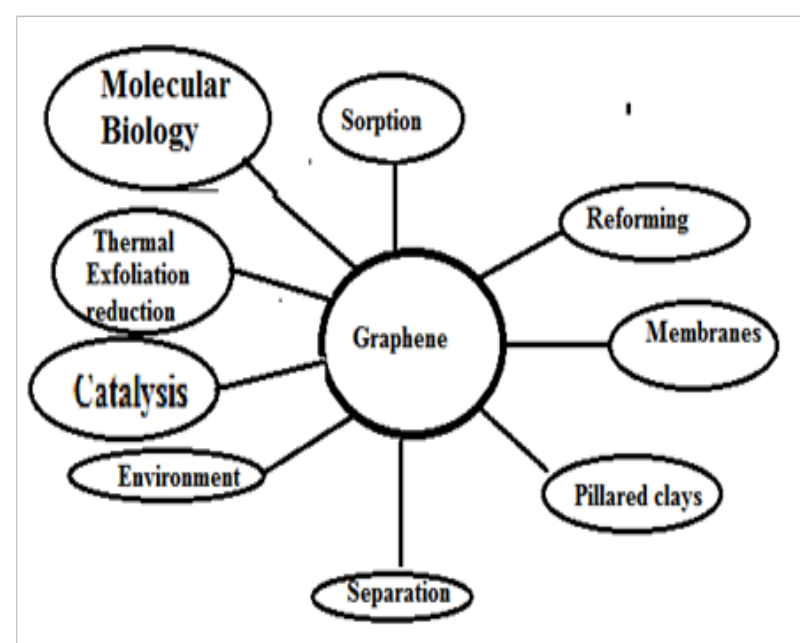

Figure 3 Graphenes utilization for different chemical processes and biological applications. $^{12}$

\section{Nanotechnology in polymers and drugs}

The polymers are very important materials, which are nanomaterials, used in different applications in the industry, reactors, chemicals and lastly in medicine or drugs, such as Polyribosomes for decease treatments, (cancer), or polymeric micelles for therapeutic applications in medicine, or polymeric vesicles/capsules for diagnostic applications, or polymeric nanoparticles for imaging in the medical area, or polymeric nanoparticles for use as carriers of drugs or other active substances such as vaccines, herbal remedies, cosmetics, or Nanoparticles for use in aqueous paints, adhesives, coatings, re-dispersible latexes, pressure sensitive adhesives, electronics, optoelectronics. ${ }^{14,15}$

To obtain Nano Capsules (NC) several processes are used, such as layer by layer self-assembly, block copolymer assembly, solvent evaporation, and polymerization in mini emulsion. The mini emulsion polymerization technique is a biphasic system, in which case the monomer and co-stabilizer are dispersed in water.

Sayer et al. ${ }^{16}$ studied polymeric carrier systems for the vectorization of hydrophobic compounds. The polymeric Nano Capsules (NCs) appears promising for controlling actives compounds in a variety of biomedical, food, cosmetics and other applications. This paper studied the encapsulation of jojoba and andiroba oils via mini emulsion polymerization. 
The drugs need to be in a biologically favorable medium for its absorption and action and the dissolution of the drug in the biological fluids is one of the main strands for an adequate bioavailability. ${ }^{17}$

\section{Biomedical nanostructures}

Nanotechnology and regenerative medicine are the great innovations of this century. ${ }^{18,19}$ The combination of these two innovative areas, through the combination of nanofibers and cells, can break several paradigms, and has been shown to be the future of regenerative organ and tissue therapy and, consequently, hope for numerous diseases such as cancer, diabetes, spinal cord injury, burns and others previously considered incurable. Biomedical nanostructures are highly promising for different applications. ${ }^{20}$

The particular cartilage is a vascular tissue containing a relatively stringers and number of chondrocyte cells that covers the joints. It is a process by which energetic contaminant ions are made to impart into silicon, which results in the penetration of the ions below the surface of the target and thus give rise to control and predictable doping distributions.)

Poly (methyl methacrylate) (PMMA) is widely used as bone cement, which is mainly used to adhere to total prosthesis rods of joints in the bone cavity. Since it acts as an interface between the prosthesis component and the bone tissue, it is necessary that this material is biologically compatible with osteoblasts (bone-forming cells) in order to improve matrix formation and the mineralization that will ultimately support osseo integration of the implant with the surrounding tissue.

Blood contact with synthetic polymer devices are widely used in blood contact implants and devices. Using vascular prostheses, for example, clinically used artificial blood vessels are most often constructed from expanded polytetrafluoroethylene (ePTFE) or polyethylene terephthalate (PET). The major concerns associated with blood contact devices are infection, thrombosis, and stenosis. ${ }^{21,22}$

Magnetic nanoparticles have received special attention. Biological application of iron oxide nanoparticles (IONPs) in magnetic resonance imaging, labeled cells, hyperthermia, immunological assays, cell separation and drugs are promising. It is important to consider the biocompatibility and toxicity of these materials. ${ }^{23}$

\section{Conclusion}

Nanotechnology is a challenge in modern engineering, changing the conventional form of teaching and research, and thus, allowing to find innovative materials and processes, based on the recent fundamental studies of inter-disciplinary areas of materials, bioprocesses and medicine, for applications on different fields in near future.

\section{Acknowledgements}

None.

\section{Conflict of interest}

The author declares no conflict of interest.

\section{References}

1. http://www.nnin.org/sites/default/files/files/scale_of_things_26may06. pdf
2. Suslick K, Taeghwan H, Mingming F, et al. Sonochemical synthesis of nanostructured catalysts. Materials Science and Engineering. 1995;204:186-192.

3. https://pt.wikipedia.org/wiki/Usu\%C3\%A 1 rio(a):Lucas Sassi/S\%C3\%ADntese_de_Materiais_Nanoestruturados.

4. RH Baughman, AA Zakhidov, WA De Herr. Carbon nanotubes--the route toward applications. Science. 2002;297(5582):787-92.

5. JP Tessonier, O Ersen, G Weinberg, et al. Selective Deposition of Metal Nanoparticles Inside or Outside Multiwalled Carbon Nanotubes. American Chemical Society Nano. 2009;3(8):2081-2089.

6. MR Loos, ESH Pezzin, ESC Amico, et al. The matrix stiffness role on tensile and thermal properties of carbon nanotubes/epoxy composites. $J$ Mater Sci. 2008;43(18):6064-6069.

7. B Astinchap, R Moradian, A Ardu, et al. Bifunctional FePt @ MWCNTs/ $\mathrm{Ru}$ Nano architectures: Synthesis and Characterization. Chem Mater. 2012;24:3393-3400.

8. Y Gao, $\mathrm{K}$ Xie, $\mathrm{S}$ Mi, et al. Preferential oxidation of $\mathrm{CO}$ in $\mathrm{H}_{2}$ rich steam over multi-walled carbon nanotubes confined Ru catalysts. Int J Hydrogen Energy. 2013;38:16665-16676.

9. WS Hummers, RE Offemann. Preparation of graphitic oxide. Journal of the American Chemical Society. 1958;80(6):1339-1339.

10. S Park, RS Ruof. Chemical methods for the production of graphenes. Nature nanotechnology. 2009;4:217-224.

11. BF Machado, P Serp. Graphene-based materials for catalysis. Catal Sci Technol. 2012;1(2):54-75.

12. S Gadipelli, Z X Guo. Graphene-based materials: Synthesis and gas sorption, storage and separation. Progress in Materials Science. 2015;69:1-6.

13. S Karimi, A Tavasoli, Y Mortazavi, et al. Cobalt supported on Graphene-A promising novel Fischer-Tropsch synthesis catalyst. Appl Catal A: General. 2015;499:188-196.

14. http://labvirtual.eq.uc.pt/siteJoomla/index.php?option=com_content\&task= view\&id=116\&Itemid=2

15. DR Paul, LM Robeson. Polymer nanotechnology: Nanocomposites. Polymer. 2008;49:3187-3204.

16. PB Cardoso, H Araujo, C Sayer. Macromol Symp. 2013;324:114-123.

17. MJ Alonso, A Sanchez, AM Campos. Chitosan Nanoparticles: a new vehicle for the improvement of the delivery of drugs to the ocular surface. Application to cyclosporine. International Journal of Pharmaceutics. 2001;1(224):159-168.

18. Kenneth E, Gonsalves. Biomedical Nanostructures. Wiley-Interscience. USA: John Wiley \& Sons Publication; 2010.

19. S Venkatraman. Has Nanomedicine lived up to its promise? Nanotechnology. 2014;25(37):372501.

20. AE Sweeney. Nanomedicine concepts in the general medical curriculum: initiating a discussion. Int J Nanomedicine. 2015;10:7319-7331

21. http://cepof.ifsc.usp.br/pesquisa/metamateriais-plasmonicos

22. 3M Science Applied to Life TM. 2016.

23. Anti-Cancer Polymersomes. 2010. 